\title{
Lectura, escritura y democracia de la cultura: experiencias con la creación literaria
}

Reading, writing and democracy of culture: experiences within literary creation

\author{
AMANDO LÓPEZ VALERO \\ Universidad de Murcia \\ ISABEL JEREZ MARTÍNEZ \\ Universidad de Castilla-La Mancha \\ España
}

\begin{abstract}
Resumen. En este artículo tratamos de mostrar una línea de pensamiento que tiene que ver con la esencialidad del uso de lo lingüístico en la construcción y desarrollo de las sociedades. Aludimos a la creación literaria, la cual, teniendo la lectura y la escritura como ejes fundamentales, posibilita que exista la democracia de la cultura. Observamos la combinación de lo instrumental y de lo cognoscitivo ya que el uso de las habilidades lingüísticas básicas puede orientarse a un uso meramente instrumental pero si unimos a esa funcionalidad una vertiente relacionada con el pensamiento y la creación, dotamos a la personas de mejores cualidades para su cotidianeidad.

La creación literaria será el medio que se muestre en este texto como exponente de la transformación social y cultural pretendida.
\end{abstract}

Palabras clave: cultura; lectura; escritura; creación literaria; aprendizaje permanente.

\begin{abstract}
It is our aim in this article to portray a line of thought related to the fundamental character of the use of language in the development of current societies. We need to take into consideration literary creation, which through the use of reading and writing allows the democracy of culture to arise. We can see the combination of instrumental abilities and different thought due to the fact that if we combine both of them we may provide people with better competences in their lives.

Literary creation will be the topic depicted in this article as a medium of social and cultural transformation. Relevant experiences will refered to, which corroborate this argument.
\end{abstract}

Key words: culture; reading; writing; literary creation; lifelong learning. 


\section{Introducción}

Los paradigmas de explicación social que en el último Siglo y en el actual hemos utilizado frecuentemente nos muestran alternativas dicotómicas que oscilan entre la predominancia del sistema donde las personas se convierten en elementos del mismo y ejecutan un papel predeterminado y la aspiración de una supremacía de las personas que son capaces de transformar el sistema social. Polémicas como la de Luhmann y Habermas han marcado las discusiones filosóficas relativas a este hecho. Al respecto Sánchez Flores (2007: 88) nos indica que:

Los planteamientos sustantivos de estas dos construcciones teóricas son opuestos, pues Habermas cree en la posibilidad moral de los individuos para construir discursiva y racionalmente la 'situación ideal del discurso', mientras que Luhmann pone por delante la realidad concreta e incontrovertible de la vida humana como constante producción de solución a problemas, la necesidad moderna de 'funcionar'. Sin embargo, aquí, argumento que el punto de partida de ambos coincide: la experiencia y autoconciencia individual moderna, en un entorno social con sentido (sustantivo en Habermas, meramente funcional en Luhmann).

Más allá de estas disquisiciones sí que apreciamos unas señas de identidad concretas del periodo postmoderno en el cual nos hallamos. Y la principal estriba en la palabra información. El fluir de la misma en una sociedad adjetivada como globalizada supone que el acceso a la misma sea el instrumento de poder al que las personas aspiran. Dicha aspiración motiva ciertos cambios sociales que afectan tanto a las estructuras sociales como a los instrumentos que soportan el entramado cultural de las citadas sociedades. Las habilidades lingüísticas también son objeto de transformación, tanto que, por ejemplo, lectura y escritura se convierten en algo instrumental y se desdeña su valor relacionado con la conservación y creación de cultura, siendo el factor fundamental que ayuden a la obtención y distribución -acreditando la mayor rapidez posible- de información.

La ciudad del lenguaje imaginada por Wittgenstein complementa, según Iriarte (2000: 236), la aportación de Luhmann:

Más allá de estas coincidencias de talante, son visibles los paralelismos entre la concepción de lo social del primero y la del lenguaje del segundo. En primer 
lugar, ambos autores comprenden sus respectivos objetos de estudio en términos de complejidad y pluralidad interna. Ni uno ni otro cree en la existencia de centros absolutos, así como tampoco en jerarquías rígidas que estructuren conforme a un orden claro y definitivo el espacio de lo social y el del lenguaje. Éste, a decir de Wittgenstein, aparece como un conglomerado de juegos que poseen sus peculiares condiciones de funcionamiento. Luhmann, por su parte, ha caracterizado lo social como una pluralidad, anárquica y heterogénea, de sistemas que poseen, asimismo, su específica lógica interna.

La insistencia de ambos en la construcción lingüística de lo social nos sirve para sustentar esta sección introductoria de esta aportación aunque en nuestro caso optaremos por una perspectiva ligeramente distante de la orientación de Luhmann y Wittgenstein quienes en sus teorías atribuyen a las personas un papel menor. Los hablantes particulares juegan muchos juegos de lenguaje y los individuos participan en múltiples sistemas. En el Wittgenstein del Tractatus el sujeto pensante forma parte de los límites del mundo; en Luhmann los sistemas psíquicos componen el entorno de la sociedad. Por lo tanto, se alejan estos pensamientos del discurso habermasiano que sí confía en una transformación social apoyada en la acción comunicativa. Tomando lo mejor de estas teorías queremos procurar una propuesta que ensalce las habilidades lingüísticas y las ponga al servicio de la ciudadanía de tal modo que la cultura sea un bien compartido por las personas y éstas tengan acceso a la misma en virtud del principio de igualdad de oportunidades.

En marzo de 2000, el Consejo Europeo de Lisboa reconoció que la adaptación a la globalización y al desplazamiento hacia las economías basadas en el conocimiento era un reto para Europa. Así, todo ciudadano debe poseer los conocimientos necesarios para vivir y trabajar en la nueva sociedad de la información y en que un marco europeo debería definir las nuevas cualificaciones básicas que deben proporcionarse a través de la formación continua: cualificaciones en materia de Tecnologías de la Información, idiomas extranjeros, cultura tecnológica, espíritu empresarial y habilidades para la socialización. Este deseo se proyecta para todas las personas tratando de favorecer una mayor participación de las personas en el desarrollo sostenible y la ciudadanía democrática.

En este texto nos centraremos en la labor educativa que tiene que ver con la creación literaria, enfocada la misma a conferir un espectro de posibilidades culturales y de pensamiento para la ciudadanía. El gran desarrollo tecnológico y social unido a la 
ideología neoliberal que se ha impuesto en los últimos tiempos motiva que sea preciso buscar soluciones para que las personas consigan un desarrollo personal óptimo y no sean meros actores de un sistema que no les permite demasiado margen de autonomía.

\section{Globalización, Estado de Bienestar y necesidad lingüística}

Desde el final de la Segunda Guerra Mundial, hemos gozado del denominado Estado del Bienestar, que si bien es cierto que ha sufrido achaques importantes, crisis (como la del año 1973 o la actual que se mantiene desde 2009), trata de aguantar estoicamente los cambios estructurales que la sociedad de la globalización quiere imponer. Si bien es la conglomeración de distintas dimensiones -ideológica, ética, cultural, jurídica, económica,...- la que origina el movimiento en un sentido u otro de la sociedad, parece que en esta agonía de la era moderna, es el factor económico el que controla las mentes de las personas (Navarro, 2000). Actualmente, la globalización implica una ausencia de señas de identidad por parte de las personas y de las sociedades, un pensamiento único dirige los designios humanos y ya no es tan distinta la labor que se realiza en una cultura que la que acontece en otra.

Aunque la situación aparente lo contrario, como nos indican Pascual y Garde (1999), el Estado del Bienestar está bien arraigado en la Cultura occidental y sus políticas constituyen una fuente insustituible de desarrollo, existiendo una percepción generalizada de que su desmantelamiento supondría un grave deterioro de la realidad económica social, por tanto no parece que debamos preocuparnos por su extinción aunque sí de su metamorfosis en algo moldeado a los designios neoliberales. Aludimos pues a la situación en la que el Estado de Bienestar permanece pero su definición es alterada en función de lo que sucede contemporáneamente. Indicábamos en la introducción que una cuestión clave de esta época tiene que ver con la información y con la perspectiva desde la cual se entiende la realidad. La palabra comodidad predomina en el desarrollo de las sociedades y ello motiva que el desarrollo y uso de las habilidades lingüísticas no sea algo relevante.

El problema surge cuando los deseos sociales no corresponden a las realidades, es decir, las premisas que se incluyen como lo deseable no se aprecian sino que se perciben situaciones contradictorias. Aunque quede un tanto lejana en el tiempo, 
debemos reseñar la declaración de la UNESCO, recogida por Delors (1996) donde se abogaba por una educación de tipo crítico, activa y creativa, que pueda hacer uso de todos los recursos posibles que se le puedan facilitar a la persona. Dicho proceso educativo debe centrarse en cuatro aspectos fundamentales del aprendizaje. Así, Aprender para saber, Aprender para hacer, Aprender para convivir y sobre todo, Aprender para ser, este aspecto incluye elementos de las otras tres dimensiones apuntadas y debe ayudarnos a la aproximación al Aprendizaje permanente. Casi quince años después las premisas se mantienen, pero, ¿se ponen en práctica? Retomando lo apuntado por Luhmann si únicamente somos partícipes de un sistema que va marcando un ritmo determinado, el actual entramado no oferta espacios para la lectura y la escritura que escapen del mero consumismo o promoción de unos determinados autores o ideas (obviamente en tiempos pretéritos esta situación también ha acontecido). El inconveniente reside en que las personas carecen de destrezas que les permitan ser críticas con estas propuestas sociales de tal manera que puedan aproximarse a la misma apreciándolas o rechazándolas desde un criterio propio.

\section{Lectura y escritura: entre la necesidad y el disfrute}

Las dos destrezas aludidas suponen el mejor activo que una persona puede tener si quiere insertarse de manera óptima en un sistema social. Las mismas se unen al resto de habilidades para propiciar que la persona posea competencia comunicativa. En lo que concierne a la necesidad de las mismas, aunque se puede sobrevivir mediante el abuso de las destrezas orales, parece evidente que existen una serie de acciones que precisan de un dominio del código escrito. Este manejo oscilará en rango que transita desde la mera cumplimentación de una instancia hasta la lectura de un texto complejo. En medio hallamos un sinfín de progresiones, y es en ellas donde hay que hacer hincapié, ya que casi todas ellas tienen que ver con la vida social activa.

El problema estriba en cómo acercar a la persona al desarrollo de estas destrezas de modo que ésta quede motivada para progresar constantemente en lo que respecta a la misma. Se puede tener un conocimiento de la lectura y la escritura que se estanca y convierte las mismas en algo de uso puntual y que no desarrolla todo el potencial de las mismas o se puede trabajar constantemente con ambas. Se puede mostrar a la persona 
que la lectura y la escritura pueden ser herramientas muy útiles en su abanico de destrezas vitales. La mejora de las dos viene dada por la evolución procesual y sobre todo por la práctica y la reflexión sobre los textos producidos y recibidos -ya que lectura y escritura no pueden disociarse en todo el proceso-. Es obvio pues que en el proceso educativo de animación y aproximación al hecho escritor la motivación hacia los tópicos de escritura y lectura y ante todo, la unión con la vida de las personas, es determinante a la hora de conseguir que el significado de la importancia de ambas sea comprendido.

El disfrute es fundamental si queremos que lectura y escritura se conviertan en dos pilares del aprendizaje permanente. El acceso a las mismas no puede ser mecánico, monótono sino que tiene que implicar una serie de connotaciones positivas para las personas. El componente motivador no puede estar ausente en cualquier acción relacionada con estas destrezas. Obviamente, la creatividad de las personas envueltas en los procesos de escritura y lectura debe ser fomentada, bien por la persona mediadora, o bien por las protagonistas de las acciones. Los pretextos deben ser hallados así como debe ser tenida en cuenta la toma de conciencia de los beneficios de las dos habilidades. La concatenación de conocimientos a veces es clave para encontrar una motivación intrínseca al uso constante de lectura y escritura. La nutrición y producción de textos y de información supone un estímulo para las personas usuarias ya que pueden comprobar cómo su realidad se expande y a la vez observan que el universo de conocimiento es muy extenso, comprobando cuestiones relativas a la historia antigua contemporánea y de igual modo, relacionadas con el futuro.

\section{La creación literaria y la democracia de la cultura}

Es evidente que no hay sociedad sin lenguaje, y por tanto, éste se convierte en elemento de vital importancia para el funcionamiento de la misma. Consideramos que el lenguaje es un vehículo que permite el desarrollo social de un colectivo, por tanto, supone un imperativo el adquirirlo de forma correcta para tener "éxito" en esta sociedad meritocrática en la que la vivimos. Más allá de consideraciones mercantilistas el dominio de la palabra supone una posición social u otra y de igual modo, una predisposición hacia determinadas situaciones. El término competencia tan usado en la 
actualidad en el ámbito educativo cobra importancia ya que anticipa el paso a la actuación, suponiendo la misma satisfacción, corrección o desgracia para la persona.

El entramado social es complejo, más de dos mil años de modernidad nos contemplan y en ella incluimos diversidad de Lenguas, textos literarios, otras tipologías textuales, artes como la pintura o el cine y en la actualidad el ámbito digital. Todo ello supone un desafío para la persona. Lo que subyace a todas estas manifestaciones culturales es su base lingüística. El trabajo con la misma facilitará la deconstrucción y comprensión de los fenómenos culturales, de lo contrario el acceso a la misma será limitado y quedará restringido a una minoría. De esta manera el supuesto Estado de Bienestar quedaría redefinido como un constructo caracterizado por la homogeneidad sin que las personas tengan la oportunidad de tener diversidad de intereses y conocimientos.

En el ámbito de la Lengua y la Literatura, encontramos la creación literaria, ésta proporciona a la persona la posibilidad de poder expresar sentimientos, inquietudes, pensamientos y sensaciones. En este momento, en la sociedad digital, prácticamente no hay cabida para el trabajo lingüístico, en este caso, creación literaria. Al contrario, paulatinamente se simplifica el valor de lo lingüístico, se elimina lo normativo y se opta por lo mínimo para dar vida al acto comunicativo. Desde esta perspectiva, procurando una alternativa, se plantea la creación literaria, centrándola en dimensiones de tipo cualitativo, comunicativo y en los supuestos del constructivismo y del aprendizaje significativo. La creación literaria implicaría el aprendizaje y desarrollo de todas las facetas comunicativas produciendo de forma activa contenidos lingüísticos y literarios.

Ejemplos clásicos del fomento de la creación literaria en la enseñanza los hallamos en las aportaciones de Rodari (1973), Rincón y Sánchez Enciso (1985) o Delmiro (2002). En ella, la persona se convierte en protagonista de su propio desarrollo, a la vez que se congratula con el resto de aprendizajes transformándose en algo bello y real. La variedad de actividades, espacios y tiempos que es demandada favorece además la comunicación, la cooperación y el descubrimiento de la imaginación en la propia vida. Desde nuestro punto de vista la persona tiene que ser partícipe de la sociedad de la cual forma parte y de la cultura que le trasciende. Para ello necesita los instrumentos necesarios para el acceso a la misma y éstos se centran principalmente en la lectura y en 
la escritura. Somos conscientes de que ambas deben ser redefinidas en función de los múltiples modos textuales que actualmente acontecen (Kress y Van Leeuwen, 2001). Así, la lectura no se limita a un texto físico sino que puede incluir nuevos formatos (i.e. digitales) y la escritura soporta también nuevos formatos. Sin olvidar pues estas cuestiones insistimos en la importancia de la creación literaria como medio de acceso a la denominada democracia de la cultura.

\section{Experiencias}

Una vez delimitada la línea argumentativa, en esta sección se trata de concretar qué se puede lograr con la promoción del uso de la creación literaria en diferentes poblaciones. En este caso, apuntaremos una experiencia realizada a nivel de ayuntamientos con personas mayores. Se trata de proporcionar a las mismas la oportunidad de mejorar sus destrezas lingüísticas mediante la creación literaria. Personas que en algunos casos no han tenido la oportunidad de alfabetizarse y por ende, de acceder en un mayor grado a la cultura mediante unas acciones continuadas en las cuales se democratiza la misma.

Los Centros Culturales Municipales se convirtieron en el medio idóneo para que las experiencias pudiesen tener lugar. Los mismos constituyen una amplia red de servicios públicos culturales extensamente descentralizados por el término municipal. Sus objetivos se basan en favorecer el acercamiento de la ciudadanía al disfrute de la cultura, el ocio y el tiempo libre, proporcionando los medios materiales y humanos necesarios para hacer esto posible y, de igual modo, propiciar el intercambio de tipo social y cultural, mediante la formación, difusión y producción cultural, interviniendo una serie de agentes intermedios: empresas de servicios, artistas, voluntariado,... Para desarrollar los dos objetivos apuntados se dicotomiza el tipo de actividades a realizar y se hace en Formativas y de Participación y promoción asociativa. Particularmente, nos interesa la primera de ellas, que estará en función de las demandas que manifieste la ciudadanía o bien como propuestas de intervención una vez detectadas las tendencias, gustos y carencias sociales.

Para la labor con las personas mayores, nuestra propuesta remitió a la creación literaria. Una de las labores principales era la de concienciar a esta población de que todavía podían hacer cosas útiles, que su ciclo vital no había concluido, que poseían y 
poseen potencialidades por descubrir, y lo más importante, que su aprendizaje puede repercutir de un modo positivo en las nuevas generaciones pertenecientes a sus familias. La pretensión era lograr que estas personas mayores que, tal vez por su contexto social, no habían podido hacerlo desarrollasen en su totalidad las manifestaciones más notables que realiza el ser humano en su transcurrir por el viaje de la vida, es decir: sentir, saber, decidir y hacer (Fullat, 1994), puede ser una gran oportunidad para estas personas el explorar las distintas posibilidades combinatorias del lenguaje.

Nuestro objetivo era el de dar una mejor utilización y correcto trato a la palabra como instrumento de comunicación. Queremos que esta población conozca el código elaborado y pueda llegar a aprenderlo haciéndolo suyo, esto lo haremos profundizando en los mecanismos del propio lenguaje y del meta-lenguaje dirigidos hacia un uso creativo, poético y lúdico de la Lengua. Intentamos conferirles un medio para desarrollar esas destrezas lingüísticas que poseen, pero que por cuestiones del contexto que les rodea no han podido llevar a cabo. Ellas mismas van a construir lo que desean saber y lo que les va a ser útil aunque con la salvedad de que esta vez sí que van a tener oportunidad de elección.

Mediante el uso de diferentes géneros literarios o de actividades que sugeridas por el alumnado del Centro Cultural se les otorga libertad para sus acciones, no les constriñe a tener que asimilar aburridas estructuras lingüísticas sino que van adquiriendo las mismas en los momentos que a ellas se les suscita la necesidad de adquirirlas. Por otra parte, es un medio de rejuvenecimiento, sólo el hecho de crear, de trabajar con algo les induce al pensamiento de que su aportación es útil, es beneficiosa, sobre todo, para ellas mismas.

A continuación exponemos un ejemplo de taller, el denominado: La novela colectiva muy acorde con lo que venimos hablando de las carencias lingüísticas de las personas mayores con privación sociocultural. Los objetivos de esta experiencia son: a) desarrollar la imaginación y la creatividad verbal y b) fomentar el trabajo cooperativo. El material preciso será austero es decir la trama marco y los personajes que el dinamizador de la actividad y el alumnado consensuen entre ellos. Respecto a las técnicas a desarrollar, se realizarán de un modo colectivo proponiendo al grupo el tema a trabajar en la novela tras poner en común las impresiones de todos los componentes. Posteriormente se perfilan los personajes y la trama marco. Se confiere a cada 
participante cuál va a ser su cometido a la hora de redactar. Se reúne el grupo para dar coherencia y cohesión a los contenidos literarios aportados. Finalmente, una vez que todos están de acuerdo con el resultado se le otorga al texto formato libro/novela. Todo ello cuanto más incentivador y ameno resulte más calará entre el alumnado y mejor será el aprendizaje, ya que, si se está contento con lo que realiza, se dará un mejor rendimiento.

Las experiencias previas nos confirman que el taller tiene una gran aceptación entre la población compuesta por personas mayores, sin ir más lejos existen publicaciones que recogen la labor de dichas personas mayores habiendo transitado por la inmersión en el taller. Por ejemplo, Nuestras voces o Tenemos la palabra. De ellos extraemos algunas producciones de las personas implicadas en la experiencia:

\section{CALMA EN LA MAR}

Abril, mes de abril, no es un mes de playa por eso está desierta por eso, esta paz por eso, esta calma.

No gritan los chiquillos, ahogando, con sus juegos el murmullo tranquilo del agua.

La mar, está en paz la mar, está en calma en esta desierta playa.

Un velero en la lejanía sobre las aguas descansa, mas una sueva brisa altera la calma, con un leve movimiento sobre sus velas blancas.

Sólo rompe esta paz sólo rompe esta calma el rugir del mar, al besar las rocas y la arena de la playa.

La mar, está en paz, la mar, está en calma 
sopla una suave brisa,

sobre las tranquilas aguas

de la mar que al chocar,

no forman olas sino encaje de

filigrana

con matices verdes y azules, color de

mar

sus crestas rizadas, rematadas en

blanco van,

tal belleza es la suya

que jamás el hombre pudiera,

tal hermosura crear.

La mar, está en paz

la mar, está en calma

su profundidad, nos habla

su profundidad, nos cuenta

mil historias, aventuras y leyendas

unas alegres, otras siniestras.

No es culpable, la mar sólo el hombre con su gran soberbia someterla y dominarla quisiera.

mas cuán necio afán

la mar, no se somete, no se domina

no se rige por humanas reglas, sólo

divinas.

La mar, está en paz

la mar, está en calma

cuán sosegada queda mi alma

en esta paz, en esta calma

es, su mejor medicina,

es, su mejor terapia.

María Pilar Díaz Nieto, Tenemos la palabra (1999:34)

\section{RECOJA MI ALMA EN VUELO}

\footnotetext{
Y vivo mi vida aquí, pero en mi amado espero recoja mi alma en vuelo y me dé refugio en Él.
} 
Sólo vivo en la esperanza de que el día que yo muera, como enamorada, tenga la alegría y el consuelo que recoja mi alma en vuelo y me dé refugio en Él.

Tan enamorada estoy, estoy tan enamorada, que sin él yo nada soy y solo espero y anhelo recoja mi alma en vuelo y dé refugio en Él.

Esta vida no es vivir, pues sólo morir yo quiero, te amo y pienso en ti y, esperando, sólo espero recoja mi alma en vuelo y me dé refugio en Él.

Dame ya la libertad, libérame de cadenas, deja que mi cuerpo muera, que abandone este suelo, recoja mi alma en vuelo y me dé refugio en Él.

Isabel Giménez Pellicer, Tenemos la palabra (1999:64)

\section{LA ESCOBA CONYUGAL}

A las afueras del hogar, ambos se miraban. En el pub, de estilo Luis XV, Doris y Rafael acariciaban la porcelana de Limoges de sus copas. Fluían las velas, sobre la mesa versallesca que los separaba.

Tras un desesperado silencio. Doris dejó un instante el café-helado, agarrando con agitado nerviosismo la mano de su esposo.

Rafael - dijo Doris-... hay una etapa de mi vida que guardé con celo, pero cada noche al acostarme, la pesadilla se me hace más latente que en ningún otro momento del 
día... A mi padre que era alcohólico, lo apodaban “el brincos” y... ¿Por qué le llamaban así? -interrumpió Rafael-.

Doris guardó silencio, miró a su alrededor, miró a su alrededor cerciorándose de no ser observada. Con tenue voz, bajando la mirada, le contestó, -saltaba los muros de las casas vecinas, violando a todo aquello que usara faldas. La policía -continuó ella-, en repetidas ocasiones, venía a casa a buscarlo. Yo tenía 6 años y recuerdo cómo era mi madre, entre sollozos, les decía que ignoraba su paradero. Al cerrar la puerta, mi madre exclamaba, ¡Dios mío, ¿a quién habrá violado ahora?- Poco estaba papá entre nosotros: solía aparecer a medianoche con delirium tremens, asaltando tambaleante el lecho donde estaba mi madre... ¡déjame, borracho..., me das asco! Esto lo escuchaba yo a través de la cortina que separaba nuestras camas. Estaba aterrada. Sabía que acto seguido vendría la respuesta ininteligible de mi padre, ¡eres mía, cacho puta, estate quieta o te ahostio! Mi madre le empujaba con el pie (o con la mano, incluso si le soplaba, caería de igual modo de bruces al suelo). Más de una vez, al levantarnos mi hermano y yo, encontrábamos al papá dormido en el suelo. Salíamos corriendo a avisar a mamá vociferando... ipapá está muerto! Ella nos tranquilizaba diciéndonos: -es un simple mareo, ya veréis que en cuanto le dé esta pastillita, se pondrá bien- ¿Qué pastilla era esa? -preguntó su marido.

Un complejo vitamínico de B12, que mi madre le colocaba debajo de la lengua.

El camarero interrumpió: ¿desean los señores tomar algo más? ¡No gracias!... respondió Rafael-, mientras Doris, absorta en su pasado, no se percató de la presencia del camarero, continuando cabizbaja. Rafael, sobrecogido por el relato y manteniendo con suave firmeza la mano de su amada esposa, y con contenida respiración... hasta que le llamó la atención la voz de la florista que conversaba en otra mesa alegremente. Sin que Doris se percatara, su marido la sacó del trance, acariciándole el sedoso rostro con dos orquídeas (fue el ramo, que cinco años atrás, llevó ella el día de su boda). Con sonrisa entrecortada, miró a su esposo, asiendo la mano que contenía las orquídeas y besándosela dijo, con sorprendente arrebato, - ¡amor mío, acabo de enfrentarme con mi pasado. Te he estado amargando con mi amargura durante estos 5 años, colocando la escoba en nuestro lecho!

La escoba representaba el muro que impidiera "brincar". Yo, la vecina de turno violada. El bordado de mi parte de sábana, mi madre... 
¡Las orquídeas..., tu amor paciente durante estos años... y mi confesión destripándome hasta este mismo instante, como por arte de magia, se esfumó!

Doris se levantó, apagando la vela sobre la mesa, se sentó sobre las rodillas de su marido, fundiéndose en un beso.

Esa noche, no apuraron el café con hielo. Raudos salieron hacia el coche y sin mediar palabra pararon en un Motel de la villa e hicieron el amor entre sábanas de "lino blanco".

A la mañana, les sorprendió la camarera con "escoba" en mano... ;oh, disculpen los Sres.!

La camarera cerró la puerta de la habitación, dejando olvidada la escoba.

Rafael y Doris en cómplice mirada, se carcajearon... y dando un salto de la cama, Doris cogió la escoba y erguida ante los ojos atónitos de su marido, en sensual strip-tease, comenzó a tararear:

¡si yo tu...vi...era u...na es...co...ba,

Si yo tuvi...e...ra una es...co...ba...

Cuántas cosas barrería!

Santy Santos García, Tenemos la palabra (1999:105-106)

\section{Conclusiones}

Tras haber argumentado la creación literaria como alternativa para que las personas puedan acceder a la cultura en igualdad de oportunidades es preciso realizar una serie de consideraciones que ayuden a valorar lo expuesto. En primer lugar, hemos podido comprobar cómo las personas mayores - colectivo escogido para mostrar las experiencias en esta aportación- todavía pueden ofrecer al resto de la comunidad su capacidad de ser personas y pueden, de igual modo, desarrollar sus habilidades. Si de veras habitamos en el Estado del Bienestar debemos realizar labores de tipo social que de un modo notable propugnen la igualdad de oportunidades entre todas las personas, siendo el fruto de la misma esa pretendida pedagogía axiológica (Quintana, 1998), relacionada con la exaltación de los valores de una sociedad, perdidos con el auge del capitalismo. El acceso a la información, en sus numerosas modalidades, supone que el 
crecimiento de la persona sea un hecho. Por ello, es fundamental el fomento de las relaciones con lo lingüístico. El dominio de lectura y escritura abrirá un espectro de posibilidades enorme para las personas y así tendrán la oportunidad de elegir.

En la época del postmodernismo en el que los elementos identificativos de una cultura no terminan de ser perfectamente definidos, dotar a las personas del uso de la palabra supone conferirles la oportunidad de conocer. Ese conocimiento les da la posibilidad de decidir si quieren transformar su realidad circundante o no. Sin embargo, si los niveles de conocimiento lingüístico son escasos, nos dirigimos a una sociedad homogénea que adolece de motivaciones ya que el conocimiento recae en un grupo reducido que decide qué ofrecer al resto de personas, las cuales reciben información filtrada y no reflexionan acerca de la misma. La situación se complica aún más cuando los referentes culturales se van perdiendo y la historia cae en el olvido, cuando no se lee y no se escribe. En ese momento la realidad percibida es tan limitada que la cercanía de la conversión en autómatas es un peligro.

El poder del lenguaje y sobre todo de su uso ha sido argumentado por autores Habermas (1987), Cummins (2002), Macedo (2005) y todos coinciden en su utilización arbitraria orientada a la adquisición de poder, pero desde estas líneas también opinamos que se puede abogar por un uso del lenguaje para el conocimiento de la realidad y el enriquecimiento social. Recordemos que determinadas funciones del mismo aluden a la expresión de sentimientos, de pensamientos o de creencias. En ese caso, no se instrumentaliza el lenguaje sino que se pone al servicio de la persona, favoreciendo la calidad de vida de la misma.

Pensamos que es pertinente que se haga un esfuerzo por dar la oportunidad de perfeccionar el lenguaje a personas que a lo largo de su vida no han tenido la oportunidad de acceder al mismo debido a distintas circunstancias, e igualmente a aquellas que lo utilizan diariamente pero que no han reflexionado acerca de su valor y sus enormes posibilidades para comprender mejor el proceso vital. 


\section{Referencias bibliográficas}

AAVV (1998). Nuestras voces. Murcia: Ayuntamiento de Murcia.

AAVV (1999). Tenemos la palabra. Murcia: Ayuntamiento de Murcia.

Cummis, J. (2002). Lenguaje, poder y pedagogía. Madrid: Morata.

Delmiro, B. (2002). Los talleres literarios como alternativa didáctica. En C. Lomas (comp.). El aprendizaje de la comunicación en las aulas (pp. 161-191). Barcelona: Paidós.

Consejo Europeo de Lisboa (2000). Conclusiones de la presidencia. http://www.europarl.europa.eu/summits/lis1_es.htm (última consulta $11 / 10 / 2010)$

Delors, J. (1996). La educación o la utopía necesaria. En Informe a la UNESCO de la Comisión Internacional sobre la Educación. La educación encierra un tesoro (pp.13-36). Madrid: Santillana/Ediciones UNESCO.

Fullat, O. (1994). Política de la Educación. Politeia-Paideia. Barcelona: Ceac.

Habermas, J. (1981). [1987]. Teoría de la Acción Comunicativa, 2 vols. Madrid: Taurus.

Iriarte, I. (2000). Sistemas autopoiéticos y juegos de lenguaje. El aire de familia entre Ludwig Wittgenstein y Niklas Luhmann. Papers, 61, 221-238.

Kress, G. y Van Leeuwen, T. (2001). Multimodal Discourse. London: Arnold.

Macedo, D. (2005). Lengua, ideología y poder. La hegemonía del Inglés. Barcelona: Graó.

Navarro, V. (2000). Neoliberalismo y estado del bienestar. Barcelona: Ariel.

Pascual, B. y Garde, J. A. (1999). El debate sobre el Estado del Bienestar: punto y seguido.

En J. A. Garde (Ed.). Políticas sociales y Estado de Bienestar en España (pp.17-44). Madrid: Trotta.

Quintana, J. M. (1998). Pedagogía axiológica. La educación ante los valores. Madrid: Dykinson.

Rincón, F. y Sánchez Enciso, J. (1985). Los talleres literarios. Barcelona: Montesinos.

Rodari, G. (1973). Gramática de la fantasía. Barcelona: Ediciones del Bronce.

Sánchez Flores, M. (2007). La filosofía del sujeto y la sociología del conocimiento en las teorías de Jürgen Habermas y Niklas Luhmann. Confines 3 (5), 87-98.

(Artículo recibido: 13-10-2010; revisado: 24-11-2010; aceptado: 26-11-2010) 